\title{
Purkinje Cell Activity in the Middle Zone of the Cerebellar Flocculus during Optokinetic and Vestibular Eye Movement in Cats
}

\author{
Tomohiro OMATA, Toshihiro KITAMA, Akihito MIZUKOSHI*, Takehiko UENO ${ }^{\dagger}$, \\ Mitsuo KAWATO ${ }^{\ddagger}$, and Yu SATO \\ Department of Physiology, *Department of Otolaryngology, and \\ ${ }^{\dagger}$ Department of Neurosurgery, Yamanashi Medical University, Tamaho, Yamanashi, 409-3898 Japan; and \\ ${ }^{\ddagger}$ ATR Human Information Processing Research Laboratories, Kyoto, 619-0288 Japan
}

\begin{abstract}
Based on the inverse dynamics theory, a previous paper reconstructed simple-spike (SS) firing rates of Purkinje cells in the cat's flocculus middle-zone by a linear-weighted summation of eye acceleration, velocity, and position during optokinetic response (OKR). The present study investigated the SS rates during combined optokinetic and vestibular stimuli of the cells recorded in the previous paper. During the sinusoidal vestibuloocular reflex (VOR) in the light (VORL) and in the dark (VORD) the firing modulation was small. During VOR suppression (VORS) by head and visual-pattern rotation in the same direction, the modulation was deep, with the peak coinciding roughly with peak ipsiversive head velocity. During VOR enhancement (VORE), the modulation was deep, with the peak coinciding roughly with peak contraversive
\end{abstract}

head velocity. If we interpret these data in relation to eye and head movements, the cells in the cat were comparable to the horizontal-gaze-velocity Purkinje cells in the monkey that encode a linear summation of eye and head velocity signals. Alternatively, if we interpret the data on the basis of the inverse dynamics theory, the SS rates during VORL, VORS, and VORE were wellfitted by the OKR components of the movements (subtraction of VORD from VORL, VORS, and VORE eye movements, respectively), but not by the whole movements, using the coefficients calculated during OKR. It is concluded that the data are interpretable by both theories when the VOR gain (eye movement/head movement) is close to 1 and the firing is dominated by eye velocity information. [Japanese Journal of Physiology, 50, 357-370, 2000]

Key words: cerebellum, eye movement, inverse dynamics, cat. In previous studies, the Purkinje cells in the cerebellar flocculus were divided into several cell types based on correlation between simple-spike (SS) activity and eye and head movements in the horizontal plane. In the monkey, the majority of recorded Purkinje cells in the flocculus and adjacent ventral paraflocculus were gaze-velocity Purkinje (GVP) cells that encode information related to gaze velocity (sum of the eye and head velocities). The horizontal GVP cells increase their SS firing rates with ipsiversive eye velocity dur- ing smooth pursuit eye movements and with ipsiversive head velocity during cancellation of the vestibuloocular reflex (VOR) by fixation of a target spot that rotates with the head. During head rotation in the dark, which elicits the VOR, the eye and head velocity components had opposite signs, and the firing rates of horizontal GVP cells were only weakly modulated $[1,2]$. A minor cell group of the recorded population in the monkey was the group of eye-movement-only cells that were sensitive only to the eye position and

Received on February 10, 2000; accepted on May 8, 2000

Correspondence should be addressed to: Yu Sato, Department of Physiology, Yamanashi Medical University, Tamaho, Yamanashi, 409-3898 Japan. Tel: +81-55-273-1111, Fax: +81-55-273-6731, E-mail: sato@res.yamanashi-med.ac.jp

Abbreviations: CD, coefficient of determination; CS, complex spike; DFT, Discrete Fourier Transform; GVP, gaze velocity Purkinje; OFR, ocular following response; OKR, optokinetic response; SS, simple spike; VOR, vestibuloocular reflex; VORD, vestibuloocular reflex in the dark; VORE, vestibuloocular reflex enhancement; VORL, vestibuloocular reflex in the light; VORS, vestibuloocular reflex suppression. 
velocity regardless of their origin [2].

In contrast, in rabbits [3-6], VOR in the dark evoked deep SS modulation of Purkinje cells in the flocculus. The modulation phase relative to the head velocity was scattered from 0 to $360 \mathrm{deg}$, and the cells were grouped into in-phase, out-phase, and intermediate-phase types [4-6]. The in-phase type, as determined during head rotation in the dark, changed to the out-phase activity pattern during head rotation in the light, and the out-phase type changed to the in-phase pattern during VOR-suppression by combined head and visual pattern rotation in the same direction [4]. Optokinetic stimuli were also effective for evoking SS modulation in the rabbit, with the modulation phase scattered from 0 to 360 deg [5,6]. De Zeeuw et al. [3] have pointed out that the rabbit flocculus has two distinct Purkinje cell groups related to horizontal- and vertical-axis head rotations, respectively. Based on the direction-selectivity of the complex-spike (CS) activity during retinal slip stimuli, they identified verticalaxis cells, which preferred horizontal to vertical stimuli. Almost all vertical-axis cells increased their SS firing with contraversive head velocity, both in the dark and in the light, with the modulation amplitude during head rotation in the light larger than that during head rotation in the dark [3]. De Zeeuw et al. [3] have suggested that GVP cells are absent in rabbits: instead, the vertical-axis cells in rabbits are comparable with the eye-movement-only cells of the monkey. Thus, there is a species difference in cell types between the monkey and rabbit, making the elucidation of the role of floccular Purkinje cells more complicated.

As for cat flocculus, Purkinje cell activity was recorded during saccadic eye movements and during head rotation in the dark [7]. Because activity during other visual and vestibular paradigms was not investigated, a detailed description of the cell type is lacking. Thus, at present, little is known about cat floccular Purkinje cell activity during combined visual and vestibular stimuli in the horizontal plane. The first aim of the present study is, following what has been done previously, to investigate further the types of Purkinje cells in relation to eye and head movements in the cerebellar flocculus middle zone, which is a functional module involved in the control of horizontal eye movement in the cat [8-12].

The second aim of the present study is to interpret the firing characteristics of the floccular Purkinje cells based on the inverse dynamics theory. The inverse dynamics theory [13] supposes that the cerebellum controls movements by computing motor dynamics information from kinematics information about the desired eye movements (see DISCUSSION for details). Following this inverse dynamics theory, Kitama et al. [14] reconstructed the time course of the SS firing frequency of Purkinje cells in the middle zone during horizontal OKR. The successful reconstruction was a weighted linear summation of eye acceleration, velocity, and position.

Kitama et al. [14] have suggested that the middle zone encodes motor dynamics information for counteracting the inertia and viscosity forces on the eye during OKR. A similar motor dynamics encoding has been demonstrated during the ocular following response (OFR) in the monkey ventral paraflocculus Purkinje cells $[15,16]$. Both OKR and OFR are evoked by the movement of a large-field visual pattern, which is a visual-modality sensory stimulus. It would be interesting to know whether the activity of a particular cerebellar zone contains motor dynamics information related to whole movements or to components of movements during multi-modal sensory stimuli. The present study examines whether the coefficients of the reconstructed SS activity, which were calculated for OKR eye movements [14], are valid for whole movements of OKR components during combined visual and vestibular stimuli.

\section{METHODS}

\section{Animal preparation and recording proce-}

dures. Three cats were used in a manner consistent with the Guidelines for Animal Experiments, Yamanashi Medical University, and also Guiding Principles for the Care and Use of Animals approved by the Council of the Physiological Society of Japan. The animal preparation and recording procedures were described in our previous paper [14]. In brief, the cat had a recording chamber implanted on the occipitoparietal bone and was placed on a turntable. Eye position was measured using a magnetic search-coil system described by Remmel [17]. A glass electrode (tip diameter $5 \mu \mathrm{m}$; resistance $1-2 \mathrm{M} \Omega$ ) filled with $2 \mathrm{M} \mathrm{NaCl}$ was used for extracellular single-unit recording of middlezone Purkinje cells. The SS activity was discriminated, and a SS density histogram was constructed.

Stimulation procedures. For optokinetic stimulation, a random dot pattern (dot diameter $0.2-1.0$ deg) was projected through a fish-eye lens onto a halfcylinder screen in front of the animal. The screen was $100 \mathrm{~cm}$ in radius and $90 \mathrm{~cm}$ in height and subtended $80 \mathrm{deg}$ horizontally and $50 \mathrm{deg}$ vertically the visual angle of the animal. The distance from the cat's eye to the screen was $100 \mathrm{~cm}$. The random dot pattern was rotated under the control of a computer. The stimulus 
velocity was monitored by an optical encoder.

For vestibular stimulation, the cat was passively rotated with a turntable driven by a servo-controlled DC motor about the vertical axis. The stimulus position was monitored by a potentiometer. Vestibular stimuli were controlled independently of optokinetic stimuli with the use of another computer. The computers were mutually connected by a parallel interface for combined optokinetic and vestibular stimuli.

During the recording of extracellular spikes of Purkinje cells, stimuli were applied using four paradigms. (1) VOR in the light (VORL): sinusoidal head rotation in the light in front of a stationary visual pattern. (2) VOR in the dark (VORD): sinusoidal head rotation in the dark. For obtaining darkness, the cat together with the turntable was covered with a shading cloth in a darkened room. (3) VOR suppression (VORS): sinusoidal head rotation with visual pattern rotation in the same direction. (4) VOR enhancement (VORE): sinusoidal head rotation with visual pattern rotation in the opposite direction. The sinusoidal stimulus pattern was adopted for maintaining the stability of unit recording. In addition, to estimate the amplitude of the VOR eye movement of each cat, the VORD paradigm was performed without recording the Purkinje cells.

During each paradigm, alertness of the cat was maintained by making a noise. In addition, caffeine and sodium benzoate $(0.2-0.3 \mathrm{mg} / \mathrm{kg})$ mixed with food were given when necessary. The VORD paradigm was problematic in that we could not judge the alertness by the appearance of the cat due to the total darkness. In the present study, we judged the alertness by characteristics of saccadic eye movements. First, in order to characterize the movements of an alert cat, saccadic eye movements were elicited by making a noise and presenting a visual target off to the side of the cat in a well-lit room. The maximum velocity was plotted against the saccade amplitude (Fig. 6A). Then, the standard deviations (SDs) of the maximum velocities in each amplitude (5-deg bin width) were calculated (Fig. 6B). If the maximum velocity of saccade was larger than the velocity corresponding to $1 \mathrm{SD}$ below the mean maximum velocity for the amplitude bin during the VORD paradigm (Fig. 6C), we considered that the alertness of the cat was maintained (i.e., Lopez-Barneo et al. [18]).

The OKR and VOR eye movements are composed of quick and slow phases. The recorded Purkinje cells sometimes had SS firing modulation in association with the quick phases. The aim of the present study was to investigate the relationship between the SS firing rates and the slow-phase eye movements. To avoid
SS activity related to the quick phases, we selected only the cycles in which quick phases did not occur for analysis. In our preliminary study we found that (1) the rate of the quick-phase occurrence increased with the stimulus amplitude, and (2) the speed of the slow phases became similar to that of the quick phases at higher stimulus frequencies, making it difficult to differentiate the two phases, in accord with the results of Donaghy [19] (see figs. 3 and 4 of Donaghy [19]). On the other hand, it was difficult to obtain quickphase-free cycles at a low frequency. For example, at a stimulus amplitude of $2 \mathrm{deg}$ (half of the peak-topeak), the quick phases were found in all cycles at a frequency of $0.125 \mathrm{~Hz}$, most cycles at $0.25 \mathrm{~Hz}$, and some cycles at higher than $0.5 \mathrm{~Hz}$. Because the use of higher stimulus frequencies makes it difficult to distinguish the slow and quick phases, we adopted a stimulus amplitude of $2 \mathrm{deg}$ (half of the peak-to-peak) and a frequency of $0.5 \mathrm{~Hz}$ in the present study. The number of quick-phase-free cycles obtained and averaged in each cell in the present study were 43.4 (mean) \pm 9.3 (SD) during VORL, 42.4 \pm 16.8 during VORS, 41.4 \pm 18.0 during VORE, and 38.1 \pm 13.6 during VORD after a total of 100-300 stimulation cycles. In addition, in some cells, a stimulus with an amplitude of 2 deg (half of the peak-to-peak) and a frequency of $1.0 \mathrm{~Hz}$ was also applied during VORD. Data analysis was performed when more than 20 quick-phase-free cycles were obtained for a single paradigm.

Data analysis. On the averaged data, the eye position signal was low-pass-filtered by a 2-pole digital Butterworth filter with a cutoff frequency of $20 \mathrm{~Hz}$ and then differentiated using 5 points for obtaining the eye velocity signal. Similarly, the eye acceleration signal was obtained by digital differentiation of the eye velocity signal. The SS density histogram was also low-pass-filtered by a 2-pole digital Butterworth filter with a cutoff frequency of $20 \mathrm{~Hz}$. The computer program was written by us using MATLAB (Mathworks).

During VORD, VORL, VORS, and VORE, in which the stimulus head velocity was sinusoidal, the amplitude and phase of the SS firing responses were analyzed using the Discrete Fourier Transform (DFT): (1) phase shift was calculated as the difference in the phase angles between the minimum of the fundamental component of the SS response and the maximum of the contralateral head position, and (2) amplitude of the SS firing modulation was calculated as half of the peak-to-peak amplitude of the fundamental component of the SS response (Fig. 3). A phase lead of $90 \mathrm{deg}$ means that the firing frequency is maximal when head velocity in the direction ipsilateral to the 
recording side is maximal. A phase lead of $270 \mathrm{deg}$ indicates that the firing frequency is maximal when the head velocity in the direction contralateral to the recording side is maximal. The amplitude of the VOR eye movement was also calculated after DFT analysis.

In our previous paper [14], based on the inverse dynamics theory, a multiple regression analysis was performed on the data obtained during OKR. The SS firing frequency (criterion variable) was reconstructed by a linear function of the eye position and its first and second time-derivatives (explanatory variables). The regression equation was given by

$$
f(t-\Delta)=a e^{\prime \prime}(t)+b e^{\prime}(t)+c e(t)+d,
$$

where $f(t), e^{\prime \prime}(t), e^{\prime}(t), e(t)$, and $\Delta$ are the SS rate at time $t$, the eye acceleration, velocity, and position at time $t$, and the time delay between firing rate and movement, respectively. Coefficients $(a, b, c)$, the constant term $(d)$, and the time delay were estimated by the ordinary least squares method. The search range for $\Delta$ was from -40 to $40 \mathrm{~ms}$. The data were averaged at a bin width of $4 \mathrm{~ms}$.

The SS activity of middle-zone Purkinje cells during the OKR, and the reconstruction of that activity with the multiple regression analysis, is reported in detail in Kitama et al. [14]. In the present study, the SS activity of some of the same units was analyzed during VORL, VORS, and VORE by seeing if the fir- ing rates were reconstructed from the slow-phase eye movements in these stimulus paradigms by the regression equation, using the coefficients that were obtained by Kitama et al. [14] from the relationship of the unit activity to the OKR eye movements. For each unit, a second reconstruction was made from the VORS, again using the coefficients obtained from the OKR, but using the parameters not of the actual eyemovement responses ("whole eye movements"), rather the OKR components of the eye movements. These were obtained by subtracting the VORD from the VORS eye movements. Similarly, the SS rates during VORL and VORE were reconstructed a second time from the OKR components of the movements. The coefficient of determination (CD), which is the square of the coefficient of correlation between the actual and reconstructed firing rates, was used as the parameter of the goodness of fit of the reconstruction. The slope of the regression line of a plot of the actual versus reconstructed SS rates was calculated. The slope should be close to 1 for a successful reconstruction set.

\section{RESULTS}

\section{Visual paradigm}

Before describing the present results, we will mention the results of the previous paper [14] in brief. The Purkinje cells in the floccular middle zone were iden-

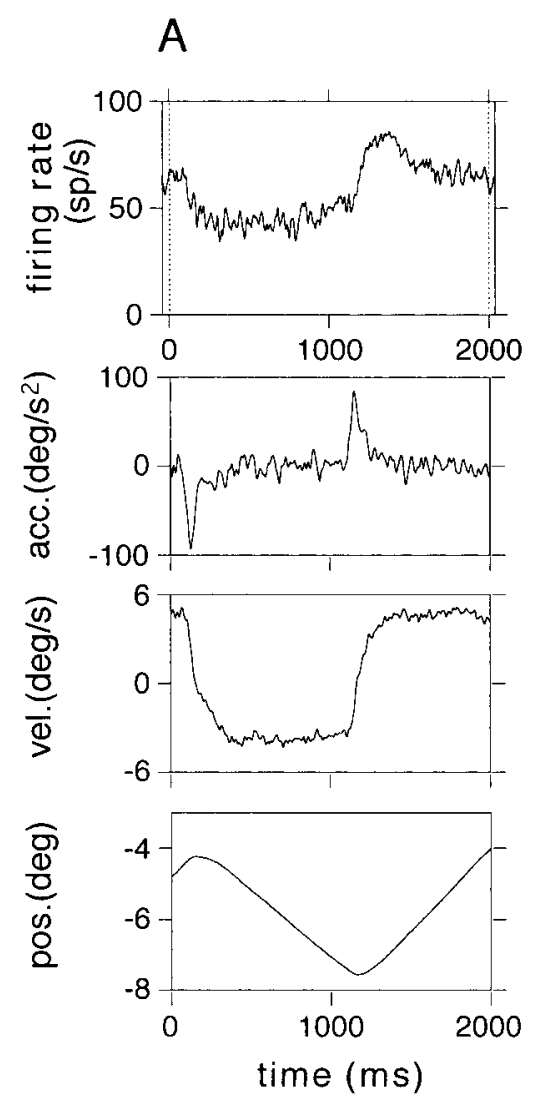

B

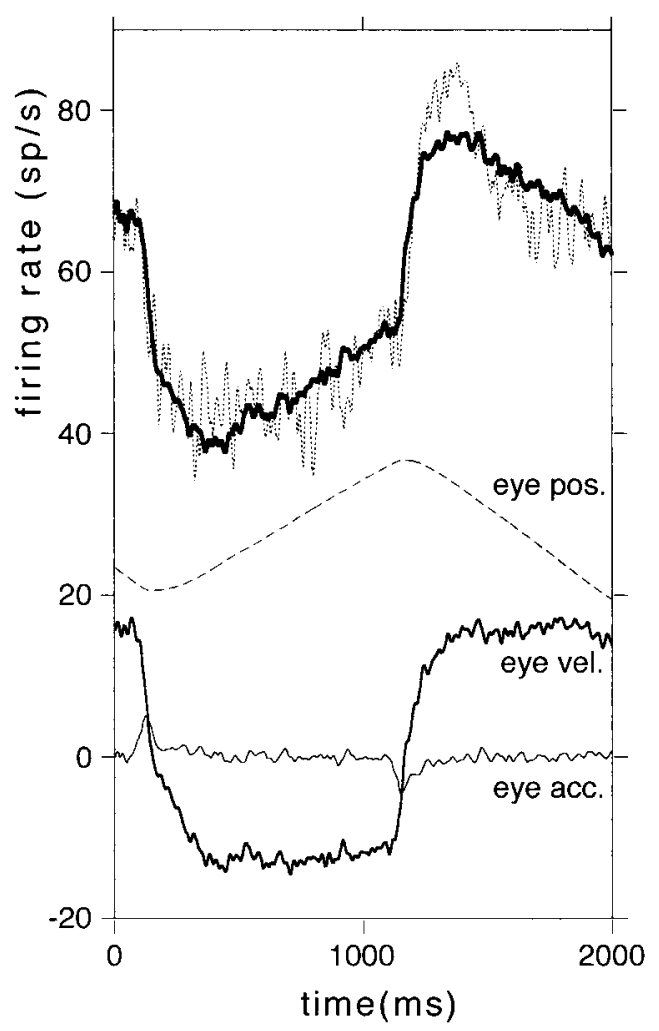

Fig. 1. Reconstruction of the simple spike (SS) firing rates of a middle-zone Purkinje cell (cell 1) during optokinetic response (OKR). A: SS firing frequency (criterion variable) and OKR eye acceleration, velocity, and position (explanatory variables). Upward deflection in the 2nd to 4th traces means position shift in the direction ipsilateral to the recording site. B: Actual (dotted line) and reconstructed (thick solid line) SS rates together with the eye position (broken line), velocity (medium solid line), and acceleration (thin solid line) components. The eye position component trace of the predicted firing rate in $\mathrm{B}$ is out-phase to the actual eye position trace in A because the eye position coefficient is negative. Data from Kitama et al. [14]. 
tified anatomically and physiologically. Anatomically, the site of location of sampled cells was specified (for details, see Fig. 1 of Kitama et al. [14]). Physiologically, the cells were identified by direction selectivity of the CS activity, which was modulated during horizontal but not vertical optokinetic stimuli. The SS firings were also modulated during horizontal OKR. A multiple regression analysis was performed to reconstruct the time course of the SS firing rates (criterion variable) with a linear function of eye position and its first and second time derivatives (explanatory variables). The regression equation was described in METHODS. Figure 1A shows the time course of the SS firing frequency (1st trace) of a middle-zone Purkinje cell (cell 1) on the left side together with the eye acceleration (2nd trace), velocity (3rd trace), and position (bottom trace), which were elicited by optokinetic velocity-step stimuli consisting of a sequence of a constant-speed $(5 \mathrm{deg} / \mathrm{s})$ visual pattern moving to the right side for $1 \mathrm{~s}$ and then to the left side for $1 \mathrm{~s}$. The velocity-step stimulus protocol was adopted to reduce the correlation between explanatory variables. The multiple regression analysis requires low correlation between explanatory variables (see Kitama et al. [14]). Figure 1B illustrates the results of the regression analysis, showing superimposed traces of the actual (dotted line) and reconstructed (thick solid line) firing rates of the cell together with the eye position (broken line), velocity (medium solid line), and acceleration (thin solid line) components. The SS rate during OKR was well fitted $(C D=0.90)$ by the reconstruction. The acceleration, velocity, and position coefficients, constant term, and time delay were
-0.055 spikes/s per deg $/ \mathrm{s}^{2}, 3.36$ spikes/s per $\mathrm{deg} / \mathrm{s}$, $-4.86 \mathrm{spikes} / \mathrm{s}$ per deg, $28.3 \mathrm{spikes} / \mathrm{s}$, and $8 \mathrm{~ms}$ (SS activity leads the eye movement), respectively. In the previous study, the coefficients for OKR eye movements were thus calculated in 41 Purkinje cells in the middle zone [14].

\section{Combined visual and vestibular paradigm}

In the present study, the firing characteristics of the Purkinje cells in the middle zone were investigated during visual and vestibular stimuli. Because it took at least $30 \mathrm{~min}$ to complete all paradigms for one cell, and because the recording sometimes became unstable during head movements, not all cells were tested with all paradigms. In 23 of the 41 cells, at least one combined visual and vestibular paradigm could be completed; that is, data from more than 20 saccade-freecycles were obtained. Of the 23 cells, all 4 paradigms were completed in 3 cells, 3 paradigms in 7 cells, 2 paradigms in 7 cells, and one paradigm in 6 cells. The data for VORL, VORS, and VORE were obtained in 15,10 , and 9 cells, respectively. In addition, VORD was tested in 12 cells at the stimulus frequency of $0.5 \mathrm{~Hz}$ and 7 cells at $1.0 \mathrm{~Hz}$. The results of VORD will be described later.

Figure 2 shows the SS firing rates and the eye movements evoked by combined visual and vestibular stimuli (sinusoidal frequency $0.5 \mathrm{~Hz}$; half of the peakto-peak amplitude $2 \mathrm{deg}$ ) in the cell from Fig. 1 (cell 1). During VORL (Fig. 2A) the head rotation in the light (3rd trace) evoked compensatory eye movements (2nd trace). The firing modulation was small (1st trace). During VORS (Fig. 2B), the head and visual-

\section{VORE}
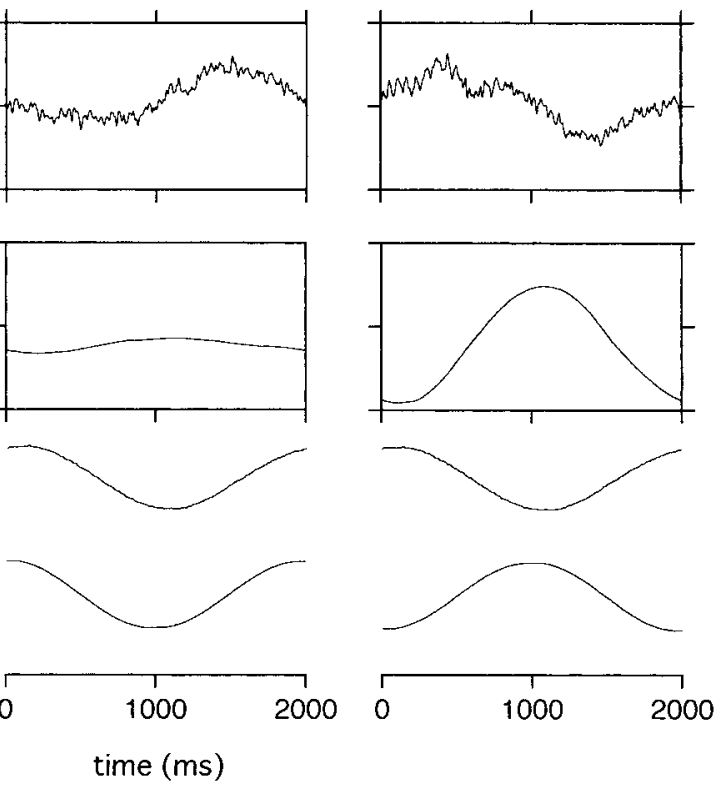

g. 2. SS firing rate (1st trace) and eye position (2nd trace) in response to combined head (3rd trace) and surround (4th trace) rotation in the cell 1. A: Vestibuloocular reflex (VOR) in the light (VORL). B: VOR suppression (VORS). C: VOR enhancement (VORE). Time 0 indicates the onset of a trigger pulse that drives a sequence of sinusoidal stimuli. Upward deflection in the 2nd to 4th traces means position shift in the direction ipsilateral to the recording site. Note that the firing modulation is small during VORL, and deep during VORS and VORE. 
A

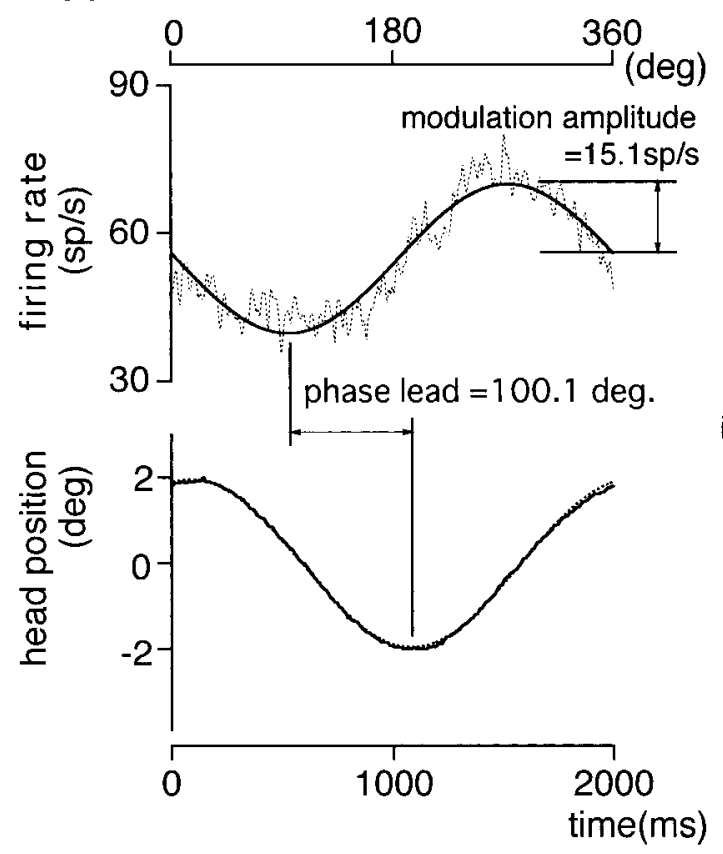

B

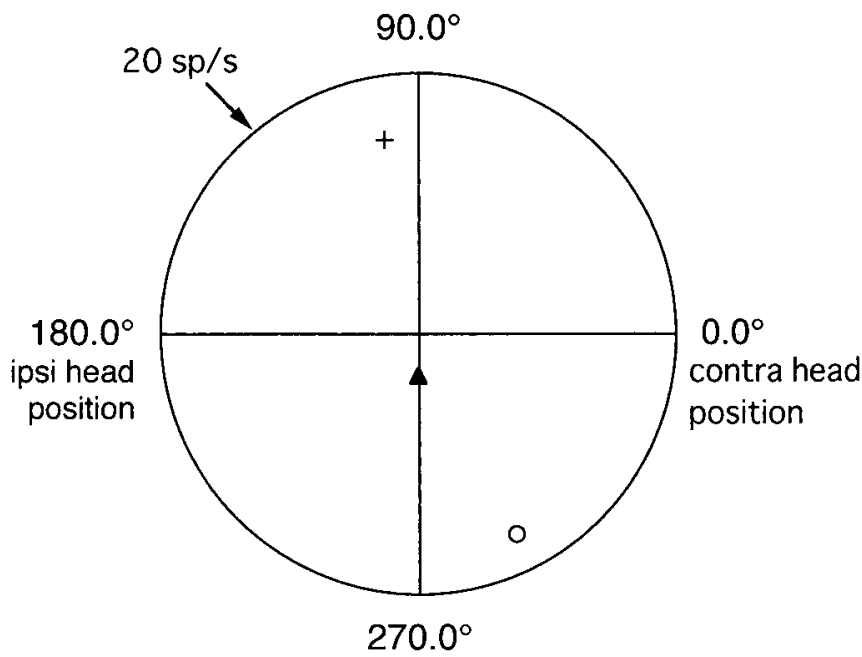

Fig. 3. Estimation of the amplitude of firing modulation tal component (solid line). Upward deflection means ipsiverand the phase relative to the head position in cell 1. A: sive position shift. B: Amplitude and phase lead during Top, SS firing rates during VORS (dotted line) and the fun- VORL (triangle), VORS (cross), and VORE (circle) were plotdamental component obtained by Fourier Transform (solid ted in polar coordinates.

line). Bottom, head position (dotted line) and the fundamen-

A VORL
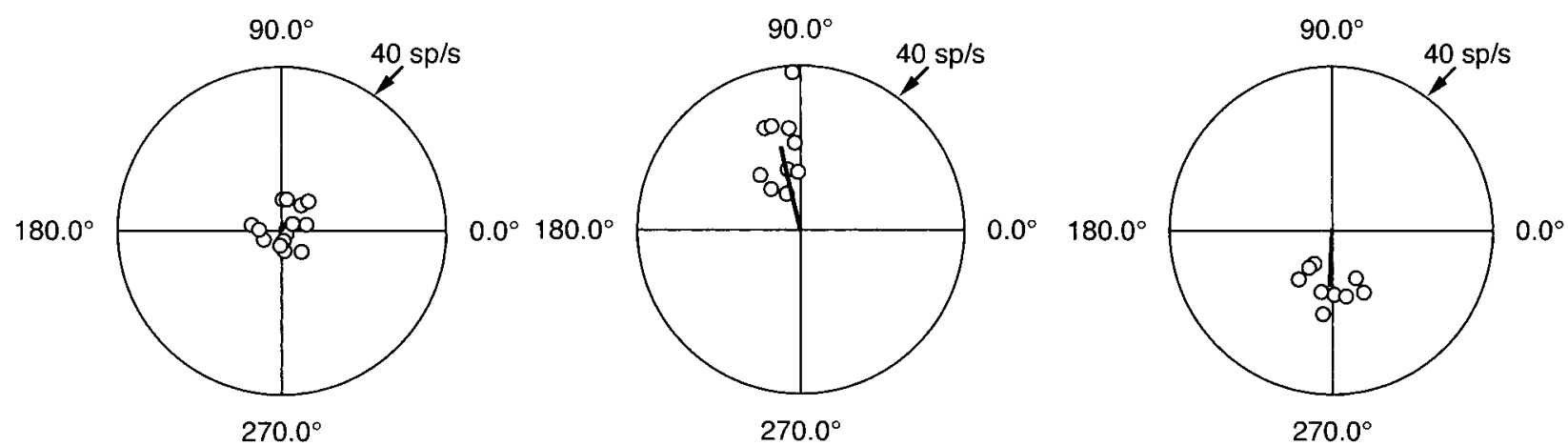

$270.0^{\circ}$

Fig. 4. Polar plots showing the modulation amplitude and phase lead of SS firing during VORL (A, $n=15$ cells), VORS (B, $\boldsymbol{n = 1 0}$ ), and VORE (C, $\boldsymbol{n}=9$ ). Note that the mean vector amplitude is close to 0 spikes/s during VORL, while relatively large during VORS and VORE, with modulation phases in opposite directions.

pattern rotation in the same direction (bottom two traces) resulted in instantaneous suppression of the VOR eye movements (2nd trace). The firing modulation was deep (1st trace). The procedures for calculating the firing amplitude and phase relative to the head position are shown in Fig. 3A. After the DFT analysis, the amplitude of SS modulation was estimated as 15.1 spikes/s with a phase lead of $100.1 \mathrm{deg}$ (see METHODS for details). These results are illustrated in the polar plot in Fig. 3B. During VORE (return to Fig. 2C), the head and visual pattern rotation in opposite directions to each other (bottom two traces) resulted in the instantaneous enhancement of VOR eye movements (2nd trace), and the firing modulation was deep (1st trace, amplitude 17.1 spikes/s) with maximum firing around the maximum head velocity in the contralateral direction (phase lead, $296.0 \mathrm{deg}$ ).

A similar tendency of the SS activity was found in all 23 cells tested (Fig. 4). That is, during VORL (Fig. $4 \mathrm{~A}, n=15$ cells), the firing modulation was small 
$A$ VORL
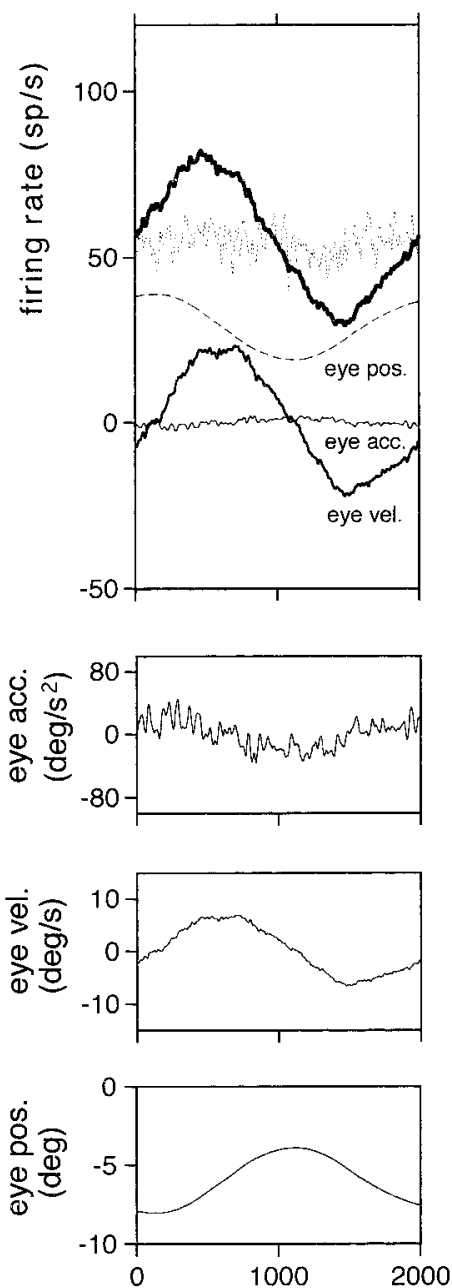

B VORS
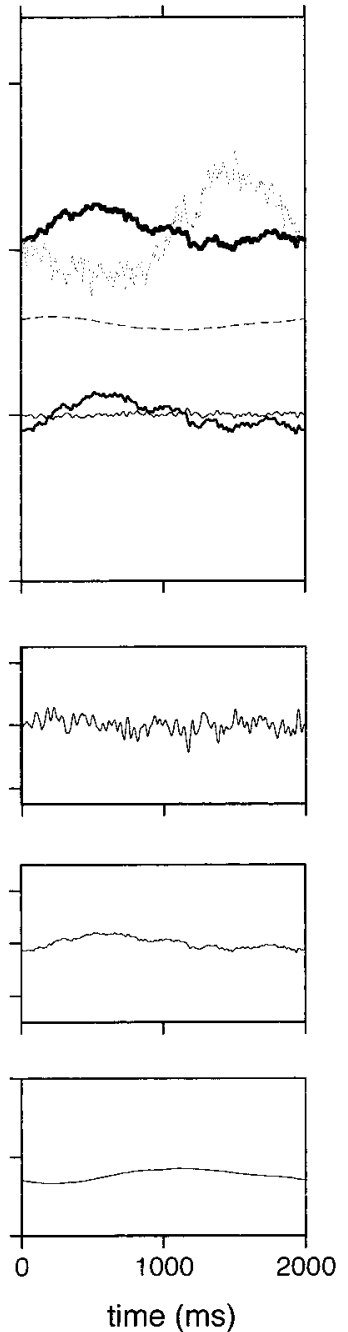

(mean vector amplitude $1.0 \mathrm{spikes} / \mathrm{s}$ ) with a scattered phase distribution pattern (mean phase lead was $46.3 \mathrm{deg}$ ). During VORS (Fig. 4B, $n=10$ cells), the modulation was large and the phase lead was around $90 \mathrm{deg}$ (mean, $20.1 \mathrm{spikes} / \mathrm{s}$ and $103.3 \mathrm{deg}$ ), indicating that firing increased during ipsiversive head velocity. During VORE (Fig. 4C, $n=9$ cells), the amplitude was large and phase lead was around $270 \mathrm{deg}$ (mean 13.7 spikes/s and $267.2 \mathrm{deg}$ ), indicating that firing increased during contraversive head velocity.

These firing characteristics of the middle-zone Purkinje cells in the cat are comparable to those of the horizontal GVP cells in the monkey $[1,2]$ (see DISCUSSION for details). In the cat, the eye position amplitude was compensatory (mean $\pm \mathrm{SD}, 2.0 \pm 0.1 \mathrm{deg}$ ), reduced $(0.8 \pm 0.2 \mathrm{deg})$, and enhanced $(3.1 \pm 0.7 \mathrm{deg})$ during VORL, VORS, and VORE, respectively.
C VORE
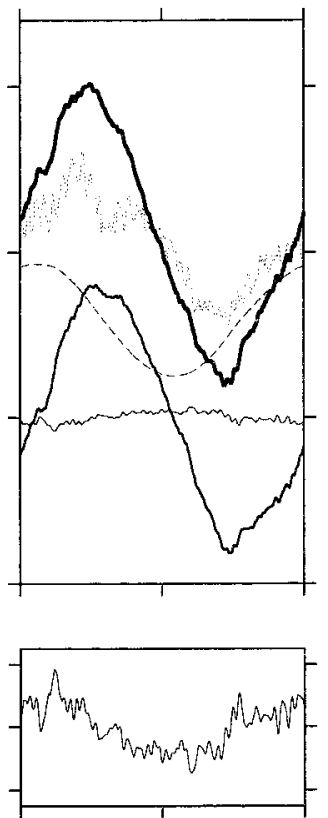

Fig. 5. Reconstruction of the SS rates during VORL (A), VORS (B), and VORE (C) from the eye acceleration, velocity and position data with the use of the coefficients calculated during OKR in cell 1. The actual (dotted line) and reconstructed (thick solid line) SS frequency together with the eye position (broken line), velocity (medium solid line), and acceleration (thin solid line) components are illustrated. Note that the reconstructed SS frequency does not fit the actual SS frequency.

\section{Interpretation of the results by the inverse dy- namics theory}

The main purpose of the present study was to investigate whether the eye-movement coefficients that were calculated during OKR [14] following the inverse dynamics theory are valid for the combined OKR and VOR eye movements. With the use of the set of coefficients calculated during OKR, reconstruction of the SS rate was made during VORL, VORS, and VORE (Fig. 5). The reconstruction was unsuccessful in all 23 cells tested. That is, the reconstructed firing modulation was large in spite of the small modulation of the actual firing during VORL (Fig. 5A). The reconstructed firing modulation was out of phase with the actual firing during VORS (Fig. 5B). And reconstructed firing overshot the actual firing during VORE (Fig. 5C). These results indicate that the coefficients for the OKR eye movements are invalid as the coefficients for the combined OKR and VOR eye movements. 
Fig. 6. Estimation of the VOR amplitude with the use of saccades as an indicator of alertness of the cat. A: Characterization of saccades of the alert cat in the light. Peak velocities of saccades were plotted against saccade amplitude when the saccades were elicited by noise or presentation of a visual target. B: Mean and SD of the peak velocities of the saccades in $A$ in each amplitude bin (bin width $=5 \mathrm{deg}$ ). C: Traces obtained during head rotation in the dark; eye velocity and eye position traces that include a saccade. The saccade has a maximal eye velocity that is greater than the lower limit of the bracketed range (mean \pm SD) in B for the appropriate amplitude bin, the criterion used for alertness. D: VOR eye movement (top trace) during head rotation (bottom trace) in the dark. Cycles were included in the averaging only if they were adjacent to cycles that contained saccades satisfying the criterion for alertness.

Previous studies have suggested that the flocculus plays an important role in visuo-motor transformation during OKR and OFR [13-16] and the time course of the SS firing during OFR was reproduced from the time course of the visual stimuli in a mathematical model [20]. Therefore, there is a possibility that the coefficients obtained from the OKR eye movement are valid only for the visually induced OKR components of the movements during combined visual and vestibular stimuli.

The OKR component of the movement is estimated by subtracting the movement induced by vestibular stimuli in the dark from the movement induced by combined visual and vestibular stimuli. The problem in estimating the VOR eye movement in the dark was that it was difficult to judge the alertness of the cat due to the darkness. The alertness was judged by the characteristics of saccadic eye movements (see METHODS for details). First, saccadic eye movements were elicited with the room lit, the maximum velocity was plotted against the saccade amplitude (Fig. 6A), and then the mean and SD for each amplitude bin (bin width $=5 \mathrm{deg}$ ) was calculated (Fig. 6B). We judged

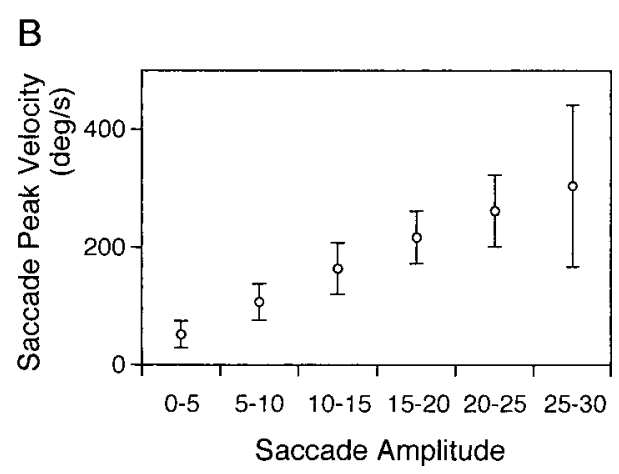

D
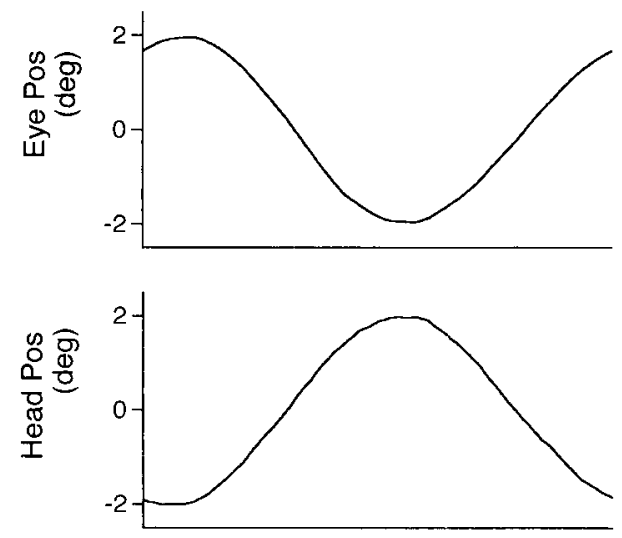

that the alertness of the cat was maintained if the maximum velocity of the saccade was more than the velocity corresponding to $1 \mathrm{SD}$ below the mean (Fig. 6B) during VORD. The VOR eye movements in the cycles immediately preceding or following such saccades (Fig. 6C) were averaged for each cat (Fig. 6D). In accord with Godaux et al. [21] and Donaghy [19], the cat exhibits little individual variability of VOR gain and phase: the VOR gain and phase lead relative to the head movement were estimated as 0.94 and $6.9 \mathrm{deg}, 0.92$ and $10.9 \mathrm{deg}$, and 0.96 and $5.6 \mathrm{deg}$, respectively, for the 3 cats used in the present study. The OKR component of eye movements (e.g., Fig. 7 bottom traces) was thus obtained by subtracting the VOR eye movement of each cat from the VORL, VORS, and VORE movements, respectively.

Theoretically, the OKR component of the SS activity should be obtained by subtracting the SS firing induced by vestibular stimuli in the dark from the SS firing induced by combined visual and vestibular stimuli. In the present study, because the SS modulation during the VORD paradigm was small (see "Vestibular paradigm" section) and not all cells were 


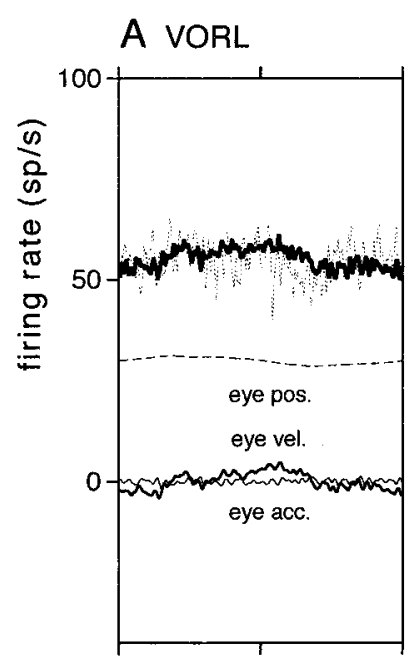

$B$ VORS
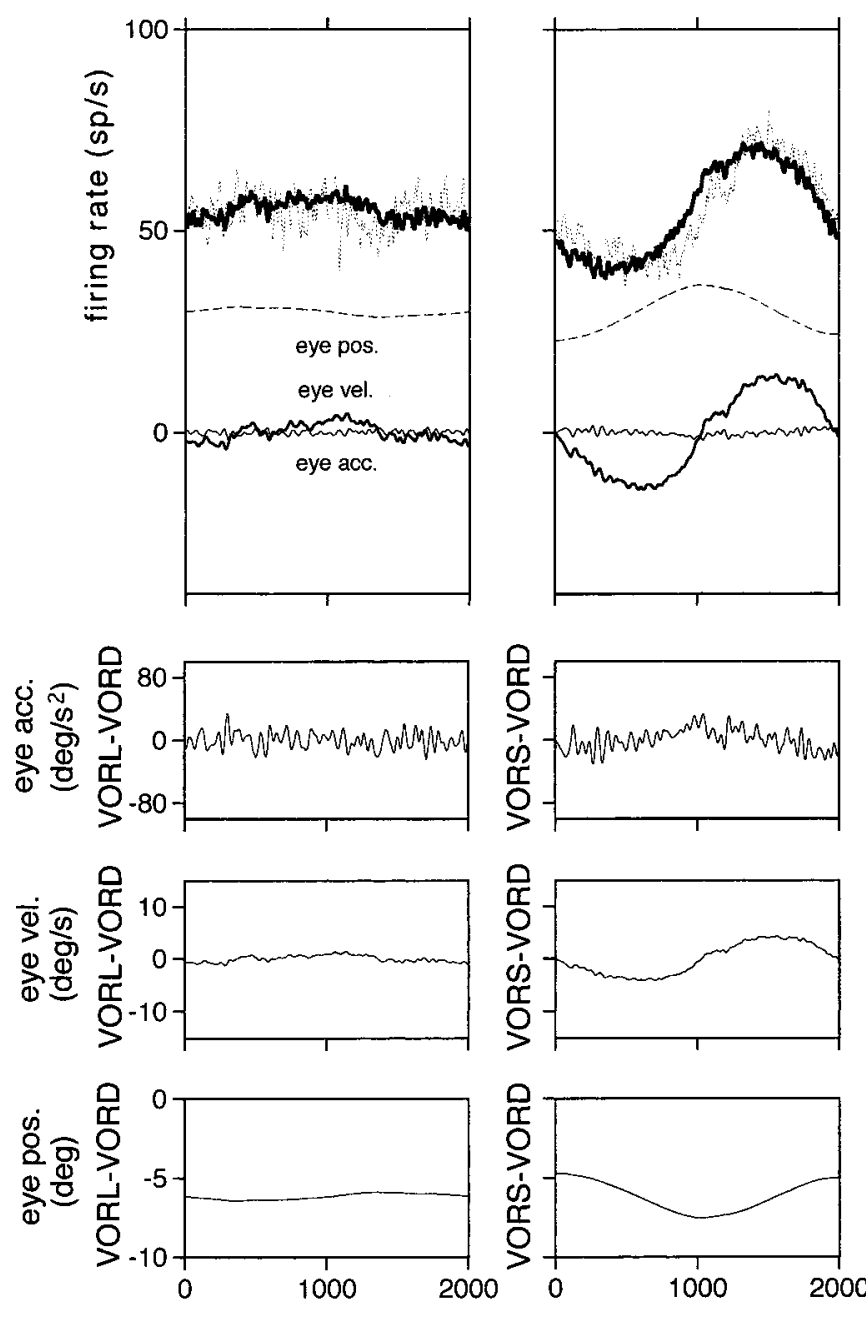

time (ms)
C VORE
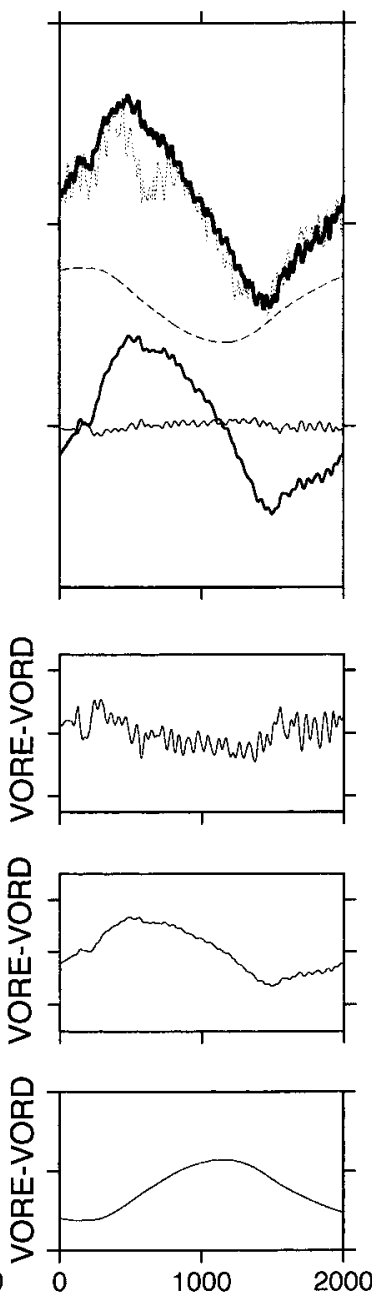

Fig. 7. Reconstruction of the SS firing rates during VORL (A), VORS (B), and VORE (C) from the OKR components of the movements (VORL-VORD, VORS-VORD or VORE-VORD) with the use of the coefficients calculated during OKR. The actual (dotted line) and reconstructed (thick solid line) SS rates, together with the eye position (broken line), velocity (medium solid line), and acceleration (thin solid line) components, are illustrated. Note that the reconstructed SS frequency fits the actual SS frequency well.

tested with the VORD paradigm, SS activity during the combined stimuli was regarded as the OKR component of the SS activity.

With the use of the set of coefficients calculated during OKR, the SS rates during VORL (Fig. 7A), VORS (Fig. 7B), and VORE (Fig. 7C) were reconstructed from the OKR components of the eye movements. The reconstructed firing frequency fit well the actual firing frequency (CDs were 0.73 and 0.89 during VORS and VORE, respectively, in Fig. 7). The correlation between the actual and reconstructed SS rates were investigated, and the slope of the regression line was 0.91 during VORS (Fig. 8A) and 0.80 during VORE (Fig. 8B), suggesting that the reconstructed firing rate overshot the actual firing frequency a little in Fig. 7B and C. The slope was similarly investigated in each cells. During VORS, the slope was distributed around 1 (mean $\pm \mathrm{SD}, 1.10 \pm 0.28, n=10$ cells). During VORE, the mean slope was $0.81 \pm 0.23$ (SD) $(n=$ cells). The mean $\mathrm{CD}$ was $0.70 \pm 0.11$ (SD) and $0.49 \pm$

\subsection{3 during VORS and VORE, respectively.}

\section{Vestibular paradigm (rotation in the dark)}

As mentioned above, during VORL, the VOR component of the movement was dominant and the SS firing modulation was small (Fig. 7A), suggesting that the motor dynamics encoding for the VOR component of movement was small in the SS firing. This was confirmed directly with the VORD paradigm. During VORD (frequency $0.5 \mathrm{~Hz}$; half of the peak-to-peakamplitude $2 \mathrm{deg}$ ), the SS firing modulation was small, while the VOR eye movement was compensatory (Fig. 9A). The mean vector amplitude was only 2.7 spikes/s and the phase lead was $124.0 \mathrm{deg}$ (Fig. 9B, $n=12$ cells). A similar tendency was found at a higher stimulus frequency of $1.0 \mathrm{~Hz}$ (half of the peak-to-peakamplitude 2 deg): mean vector amplitude was 0.86 spikes/s and the phase lead was $355.3 \mathrm{deg}$ (Fig. 9C, $n=7$ cells). 
A VORS

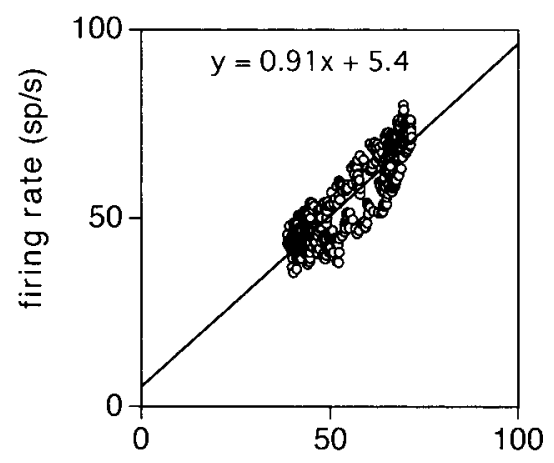

B VORE

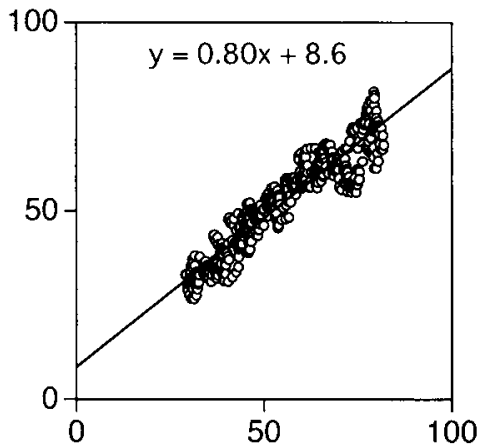

reconstructed firing rate $(\mathrm{sp} / \mathrm{s})$

A
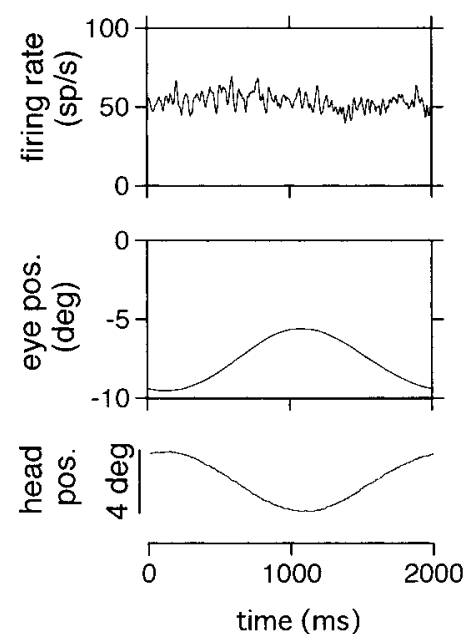

B $0.5 \mathrm{~Hz}$

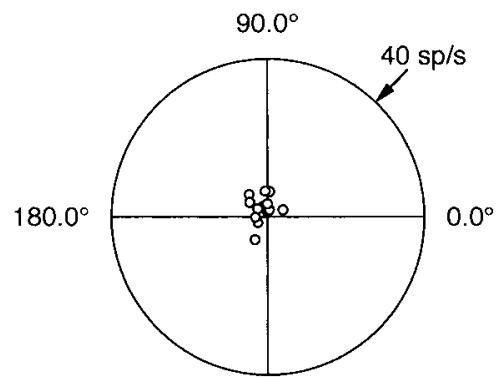

$270.0^{\circ}$
Fig. 8. Actual SS firing rates during VORS (A) and VORE (B) plotted against the reconstructed rates of cell 1 . The firing rates are from the data illustrated in Fig. 7B and $\mathrm{C}$. Each point represents the rate from one time bin of the stimulus cycle. The regression line of the actual rate on the reconstructed rate is shown.

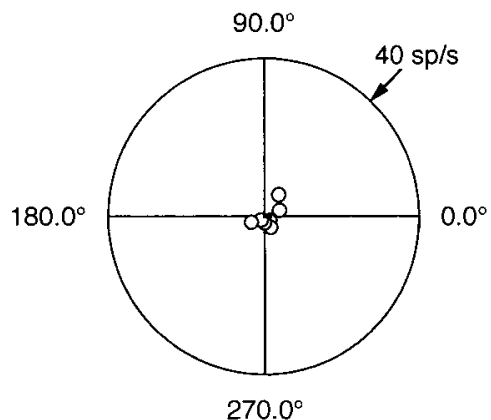

Fig. 9. VORD paradigm. A: SS firing rates (1st trace) and eye position (2nd trace) during sinusoidal $(0.5 \mathrm{~Hz})$ head rotation (3rd trace) in the dark in a Purkinje cell. B and C: Polar diagrams showing the modulation amplitude and phase lead of SS firing during VORD at stimulus frequencies of $0.5 \mathrm{~Hz}(\mathrm{~B}, n=12$ cells) and $1.0 \mathrm{~Hz}(\mathrm{C}, n=7$ cells). Note that the firing modulation is small.

\section{DISCUSSION}

Firing characteristics in relation to eye and head movements. In this section, we discuss the previous and present results based on the firing characteristics in relation to eye and head movements. As mentioned in the introduction, there is a species difference in the types of floccular Purkinje cells found in the monkey and rabbit. In the monkey, the majority of recorded cells consisted of GVP cells that encode a sum of the eye and head velocities. The gaze velocity theory says that these cells carry a signal for gaze velocity [1,2]. In contrast, in the rabbit, De Zeeuw et al. [3] suggested that the majority of cells were comparable with eye-movement-only cells, a minority group of cells in the monkey.

The present study, in addition to the previous study
[14], investigated the SS firing characteristics of the middle-zone Purkinje cells in the cat during visual and vestibular stimuli. During the OKR paradigm, in which the head velocity input is absent, the SS firing increased when the eye movement was directed ipsilaterally to the recording site, and the contribution of the velocity term to the SS firing modulation was predominant over the acceleration and position terms (Fig. 1 and Kitama et al. [14]). During the VORS paradigm, in which the eye velocity is small in amplitude, SS firing increased with ipsiversive head velocity (Figs. 2-4). During the VORL paradigm, in which the eye and head velocity components had opposite signs, the SS firing rates were only weakly modulated (Figs. 2-4). These firing characteristics of the middlezone Purkinje cells in the cat were thus comparable to those of the GVP cells in the monkey. Unfortunately, 
in the cat, the suppression of VOR eye movement by head and visual pattern rotation in the same direction was incomplete (Fig. 2B), and the measurement of head velocity sensitivity was difficult during the VORS paradigm.

A previous study using the cat reported that the majority of recorded Purkinje cells consisted of "vertical eye velocity cells" comparable to the monkey's eyemovement-only cells, which increased SS rates during, e.g., upward rotation of the head and downward rotation of the OKR visual pattern [22,23]. Part of the difference between the present and previous results may be explained by a difference in the procedures used in selecting Purkinje cells. Fukushima et al. $[22,23]$ selected only Purkinje cells that responded to pitch rotation of the head, and they reported a number of Purkinje cells that were not sensitive to head pitch rotation. On the other hand, we identified horizontaltype Purkinje cells in the middle zone by CS activity that was modulated during horizontal but not vertical retinal-slip stimuli. The majority of the recorded cells in the middle zone showed such CS direction selectivity [14]. De Zeeuw et al. [3] also identified verticalaxis (horizontal movement) Purkinje cells by direction-selective CS responses during retinal slip in the rabbit. Furthermore, the difference between the present and previous [23] results regarding a GVP versus an eye-movement-only pattern of discharge may be explained by different processing of the horizontal and vertical eye movements by the flocculus. Fukushima et al. [24] reported that the population of vertical- and horizontal-type Purkinje cells in the monkey flocculus had markedly different discharge properties, and the vertical GVP cells belong to a minority cell group in vertical-type Purkinje cells. If a similar situation exists in the cat, it would explain why the vertical units recorded by Fukushima et al. [23] showed predominantly eye-movement-only activity while the horizontal units we recorded showed the GVP pattern.

Interpretation of SS firing characteristics based on the inverse dynamics theory. In

this section, we interpret the present findings based on the inverse dynamics theory, which was proposed to explain the neural mechanisms controlling human movements with quick and smooth characteristics [13]. Usually, robot movements can be exquisitely controlled by a feedback loop that measures the difference between desired and actual movements. In humans, however, it takes a long time (50-200 ms) for a feedback loop to transmit signals due to sensor dynamics, slow conduction time of axons and the synaptic delay, suggesting that the slow feedback loop is not adequate to control quick and smooth movements in humans. Human movements may be controlled by the feedforward neural circuits that predict forthcoming movements based on present sensory information. Kawato et al. [13] proposed that the inverse dynamics model is necessary for feedforward control. The inverse dynamics model calculates the necessary motor command based on the desired trajectory, while the forward dynamics model calculates the trajectory based on the motor command information. The inverse dynamics model has been suggested to exist in the cerebellum on the basis of the findings that the cerebellar Purkinje cells encode motor command information (elastic, viscous, and inertia forces related to eye position, velocity, and acceleration, respectively) during OFR and OKR [14-16]. If the inverse dynamics theory is true, the Purkinje cell should also encode motor command information in its firing pattern during combined visual and vestibular movements.

In the present study, the movements during VORL, VORS, and VORE are composed of two components, the OKR and VOR components, because the eye movements are induced by combined OKR and VOR stimuli. The OKR-component of the movement was obtained by subtracting the VOR component (VORD movement) from the whole movement (VORL, VORS or VORE movement). The reconstruction of the SS firing frequency during VORL, VORS and VORE was made using the coefficients for OKR. The reconstruction was successful when it used the OKR component of eye movement (Figs. 7 and 8) but not when it used the whole eye movement (Fig. 5), suggesting that the coefficients calculated during OKR are valid only for the OKR component of the movement, and not for the whole movement induced by combined visual and vestibular stimuli. The coefficients calculated during a given stimulus modality may be valid only for that modality, and not for a different modality. In the cat, the brainstem pathway (i.e., Uchino et al. [25]) may be enough for generating compensatory VOR. The present study has, for the first time, shown that the firing property explanation based on the inverse dynamics theory is valid not only during visually induced movements but also during combined visual and vestibular movements. A given cerebellar zone may control some (but not all) components of the complex movements.

Comparison between the inverse dynamics and gaze velocity theories. The present study has shown that the SS firing characteristics of the middle-zone Purkinje cells in the cat are comparable to those of the GVP cells in the monkey and also interpretable by the inverse dynamics theory. We explain 
the reason why the data were interpretable by both the gaze velocity and inverse dynamics theories in the APPENDIX. The conclusion in the APPENDIX is that the SS firing modulation during combined visual and vestibular stimuli can be explained almost equally by both theories if the VOR gain (eye movement/head movement) is close to 1 , and if the Purkinje cell firing is dominated by the eye velocity signal (see APPENDIX for details). The present study has shown that the gain of VOR is close to 1 during VORD in the cat (Fig. 6), in agreement with the studies of Godaux et al. [21] and Donaghy [19]. Kitama et al. [14] have shown that Purkinje cell firing is dominated by the eye velocity signal in the middle zone of the cat.

The SS firing modulation was small in amplitude during VORL, in which the VOR component of the movement was dominant (Fig. 4A). SS modulation was also small during VORD (pure VOR) eye movement (Fig. 9). According to the inverse dynamics theory, these findings are simply interpretable as meaning that the Purkinje cells do not encode motor dynamics information for the VOR components of eye movement. In other words, the floccular Purkinje cells may not be responsible for calculation of the motor dynamics information from the vestibular modality information (head-movement information).

It should be stressed that this conclusion is valid only for the cat and the monkey, whose VOR gains are close to 1 . In the rabbit, the VOR gain is substantially lower than 1 (i.e., gain $=0.25-0.31$ by De Zeeuw [3]), and the floccular Purkinje cells are deeply modulated during VOR in the dark [3-6]. This modulation is interpretable, based on the inverse dynamics theory, as encoding motor dynamics information that is calculated from the head-movement information. In the rabbit, in which VOR eye movement is less compensatory, both the brainstem pathway and flocculus would be responsible for generating VOR eye movements. Thus, it is possible to explain the firing characteristics in the rabbit, whose VOR gain is less than 1, by the inverse dynamics theory. It would be of great interest to investigate, using the inverse dynamics theory, the motor dynamics information encoded in Purkinje cells during eye movements elicited by combined visual and vestibular stimuli in the rabbit flocculus.

According to the inverse dynamics and gaze velocity theories, eye velocity information is encoded in the SS firing rates. The most crucial difference between the two theories lies in their views about the origin of the eye velocity information encoded in the firing rates. According to the inverse dynamics theory, motor dynamics information (including eye velocity information) is calculated from kinematics informa- tion that is obtained via the sensory input. Therefore, the eye-movement coefficients are specific to the input modality. In marked contrast, in the gaze velocity theory, eye velocity information is supposed to be an efference copy of the motor command, and, therefore, the eye velocity coefficient should be generally applicable to all kinds of eye velocities regardless of the input modality. For example, according to the gaze velocity theory, an eye velocity coefficient that was calculated during smooth pursuit eye movement should also be considered valid for VOR eye movement. The small modulation of SS firing during VOR was interpreted, according to the gaze velocity theory, as the cancellation of the SS modulation of eye-velocity-sensitivity origin by the SS modulation of headvelocity-sensitivity origin $[1,2]$. However, at present, the eye-velocity sensitivity of the monkey GVP cells calculated during smooth pursuit eye movement has not been proven to be valid during VOR or OKR. We hope that future studies will further clarify the relative merits of these theories and thereby lead to a better understanding of cerebellar motor control mechanisms.

We thank Dr. David W. Sirkin for comments on the manuscript, and Prof. Hideaki Nukui, M.D. and Prof. Yoshitaka Okamoto, M.D. for providing facilities for the research. This research was supported by a grant from the Ichiro Kanehara Foundation.

\section{REFERENCES}

1. Lisberger SG and Fuchs AF: Role of primate flocculus during rapid behavioral modification of vestibuloocular reflex. I. Purkinje cell activity during visually guided horizontal smooth-pursuit eye movements and passive head rotation. J Neurophysiol 41: 733-763, 1978

2. Miles FA, Fuller JH, Braitman DJ, and Dow BM: Longterm adaptive changes in primate vestibuloocular reflex. III. Electrophysiological observations in flocculus of normal monkeys. J Neurophysiol 43: 1437-1476, 1980

3. De Zeeuw Cl, Wylie DR, Stahl JS, and Simpson Jl: Phase relations of Purkinje cells in the rabbit flocculus during compensatory eye movements. J Neurophysiol 74: 2051-2064, 1995

4. Ghelarducci B, Ito M, and Yagi N: Impulse discharges from flocculus Purkinje cells of alert rabbits during visual stimulation combined with horizontal head rotation. Brain Res 87: 66-72, 1975

5. Nagao S: Eye velocity is not the major factor that determines mossy fiber responses of rabbit floccular Purkinje cells to head and screen oscillation. Exp Brain Res 80: 221-224, 1990

6. Nagao S: Contribution of oculomotor signals to the behavior of rabbit floccular Purkinje cells during reflex eye movements. Neurosci Res 12: 169-184, 1991

7. Cheron G, Dufief MP, Gerrits NM, Draye JP, and Go- 


\section{Motor Dynamics Encoding in Cerebellum}

daux E: Behavioural analysis of Purkinje cell output from the horizontal zone of the cat flocculus. Prog Brain Res 114: 347-356, 1997

8. Sato $Y$, Kanda $K$, and Kawasaki T: Target neurons of floccular middle zone inhibition in medial vestibular nucleus. Brain Res 446: 225-235, 1988

9. Sato $Y$ and Kawasaki T: Operational unit responsible for plane-specific control of eye movement by cerebellar flocculus in cat. J Neurophysiol 64: 551-564, 1990

10. Sato $Y$ and Kawasaki T: Identification of the Purkinje cell/climbing fiber zone and its target neurons responsible for eye-movement control by the cerebellar flocculus. Brain Res Rev 16: 39-64, 1991

11. Sato $Y$, Kawasaki T, and Ikarashi K: Zonal organization of the floccular Purkinje cells projecting to the vestibular nucleus in cats. Brain Res 232: 1-15, 1982

12. Sato $\mathrm{Y}$, Kawasaki $\mathrm{T}$, and Mizukoshi K: Eye movement control by Purkinje cell/climbing fiber zones of cerebellar flocculus in cat. Acta Otolaryngol Suppl (Stockh) 481: 237-241, 1991

13. Kawato M, Furukawa K, and Suzuki R: A hierarchical neural-network model for control and learning of voluntary movement. Biol Cybern 57: 169-185, 1987

14. Kitama T, Omata T, Mizukoshi A, Ueno T, and Sato Y: Motor dynamics encoding in cat cerebellar flocculus middle zone during optokinetic eye movements. J Neurophysiol 82: 2235-2248, 1999

15. Gomi H, Shidara M, Takemura A, Inoue $Y$, Kawano K, and Kawato M: Temporal firing patterns of Purkinje cells in the cerebellar ventral paraflocculus during ocular following responses in monkeys i. simple spikes. J Neurophysiol 80: 818-831, 1998

16. Shidara M, Kawano K, Gomi H, and Kawato M: Inverse-dynamics model eye movement control by Purkinje cells in the cerebellum. Nature 365: 50-52, 1993

17. Remmel RS: An inexpensive eye movement monitor using the scleral search coil technique. IEEE Trans Biomed Eng 31: 388-390, 1984

18. Lopez-Barneo J, Ribas J, Delgado-Garcia, and Mir D: Effects of drugs on the characteristics of cat's saccadic eye movements. Neuurosci Lett 8: 347-353, 1978

19. Donaghy M: The cat's vestibulo-ocular reflex. J Physiol (Lond) 300: 337-351, 1980

20. Yamamoto K, Kobayashi Y, Takemura A, Kawano K, and Kawato $\mathrm{M}$ : A mathematical model that reproduces vertical ocular following responses from visual stimuli by reproducing the simple spike firing frequency of Purkinje cells in the cerebellum. Neurosci Res 29: 161-169, 1997

21. Godaux E, Gobert E, and Halleux J: Vestibuloocular reflex, optokinetic response, and their interactions in the alert cat. Exp Neurol 80: 42-54, 1983

22. Fukushima K, Buharin EV, and Fukushima J: Responses of floccular Purkinje cells to sinusoidal vertical rotation and effects of muscimol infusion into the flocculus in alert cats. Neurosci Res 17: 297-305, 1993

23. Fukushima $K$, Chin S, Fukushima J, and Tanaka M: Simple-spike activity of floccular Purkinje cells responding to sinusoidal vertical rotation and optokinetic stimuli in alert cats. Neurosci Res 24: 275-289, 1996

24. Fukushima K, Fukushima J, Kaneko CR, and Fuchs AF:
Vertical Purkinje cells of the monkey floccular lobe: simple-spike activity during pursuit and passive whole body rotation. J Neurophysiol 82: 787-803, 1999

25. Uchino $Y$, Hirai N, and Suzuki S: Branching pattern and properties of vertical- and horizontal-related excitatory vestibuloocular neurons in the cat. J Neurophysiol 48: 891-903, 1982

\section{APPENDIX}

In the present study, the slow-phase eye movement $(E)$ relative to the head is assumed to be composed of a linear summation of the VOR-component eye movement $\left(E_{\mathrm{VOR}}\right)$ and the OKR-component eye movement $\left(E_{\mathrm{OKR}}\right)$.

$$
E=E_{\mathrm{VOR}}+E_{\mathrm{OKR}} \text {. }
$$

Because the VOR gain is close to 1 in the cat, $E_{\mathrm{VOR}}$ is of the same amplitude as the head movement $(H)$ and of the opposite sign.

$$
E_{\mathrm{VOR}}=-H \text {. }
$$

From Eqs. 1 and 2,

$$
E=-H+E_{\mathrm{OKR}} .
$$

The gaze position $(G)$ in the earth-fixed coordinates is the sum of $E$ and head position $(H)$

$$
G=E+H \text {. }
$$

From Eqs. 3 and 4

$$
G=\left(-H+E_{\mathrm{OKR}}\right)+H=E_{\mathrm{OKR}} .
$$

During combined VOR and OKR movements, the motor command $(M)$ becomes a linear addition of the VOR-component motor command $\left(M_{\mathrm{VOR}}\right)$ and the OKR-component motor command $\left(M_{\mathrm{OKR}}\right)$, if the motor plant dynamics are assumed linear.

$$
M=M_{\mathrm{VOR}}+M_{\mathrm{OKR}} \text {. }
$$

The present study has provided evidence that middlezone Purkinje cell firing $(P)$ is related to $M_{\mathrm{OKR}}$, and actually constitutes a part of $M_{\mathrm{OKR}}, M_{\mathrm{OKR}}{ }^{P} . P$ is reconstructed from $E_{\mathrm{OKR}}$, and its first $\left(E_{\mathrm{OKR}}{ }^{\prime}\right)$ and second $\left(E_{\mathrm{OKR}}{ }^{\prime \prime}\right)$ time derivatives by the equation:

$$
P=M_{\mathrm{OKR}}{ }^{P}=a E_{\mathrm{OKR}}{ }^{\prime \prime}+b E_{\mathrm{OKR}}{ }^{\prime}+c E_{\mathrm{OKR}}+d .
$$

The analysis performed using the standard regression coefficient showed that the contribution of the velocity term to the regression is dominant over the acceleration and position terms [14]. If we ignore the acceleration and position terms, $P$ is approximated by:

$$
P=b E_{\mathrm{OKR}^{\prime}}+d \text {. }
$$


From Eqs. 5 and 8,

$$
P=b G^{\prime}+d .
$$

On the other hand, according to the gaze velocity theory $[1,2]$, floccular Purkinje cell firing modulation represents the gaze velocity $\left(G^{\prime}\right)$.

$$
P=b G^{\prime}+d .
$$

Equation 10, which is based on the gaze velocity theory, is the same as Eq. 9, which is the approximation of Eq. 7 based on the inverse dynamics theory. This suggests that if the VOR gain is close to 1 , and if Purkinje cell firing is dominated by velocity information, the experimental data concerning Purkinje cell firing during combined OKR and VOR can be interpreted approximately equally well by both theories. 